\title{
Drift patterns in an Antarctic channel from a quasi- geostrophic model with surface friction
}

\author{
JÖrg-Olaf WolfF, John A.T. Bye ${ }^{2}$ \\ ${ }^{1}$ Antarctic CRC, Box 252-80, Hobart, Tasmania 7001, Australia \\ ${ }^{2}$ School of Earth Sciences, The Flinders University of South Australia, Box 2100, Adelaide, South Australia 5001, Australia
}

\begin{abstract}
The surface layer of the Southern Ocean is subject to the action of wind, waves and currents. We present solutions from a fine-resolution quasi-geostrophic model with surface friction, which is driven by a specified mean and fluctuating wind field, and predicts the surface current, and also the surface Stokes drift due to the wavefield. The resulting flow patterns control the dispersion of particles at the sea surface, and, using a proven Lagrangian algorithm, batches of particles of specified draught can be injected into the flow at various locations and tracked. The simulated patterns are compared with historical data on dispersion and with drift-card and satellite-drogue studies in the Southern Ocean, iceberg tracking and other studies to show the relative importance of dispersion by synoptic variability in the atmosphere and mesoscale eddies in the ocean.
\end{abstract}

\section{INTRODUCTION}

The purpose of this paper is to indicate the similarities and differences to be expected in the dispersion patterns for material in the surface layer of the ocean in an Antarctic channel. The Southern Ocean is unique in so far as it forms a global annulus around which oceanic properties take on an almost zonal character, which is essentially due to advection by currents generated by the predominantly westerly wind circulation. Superimposed on this climatological regime are the synoptic weather systems in the atmosphere, and the mesoscale eddies in the ocean.

This time-varying circulation gives rise to dispersive processes, which are controlled by the surface geostrophic wind and current, and by the wavefield. We consider the response over scales extending from surface films, through objects which comprise flotsam, either natural (e.g. decaying tree trunks), or anthropogenic (e.g. buoys or containers which fall off freighters), to deep draughted objects such as icebergs.

The flow fields are generated by a quasi-geostrophic ocean model, which is coupled to the atmosphere by a surface stress relation which explicitly incorporates the wavefield. Using this model, it is possible to simulate the dispersion of a variety of material of specified draught.

\section{THE MODEL}

The fine-resolution two-layer quasi-geostrophic model was originally developed in Wolff and others (1991), and the surface stress coupling with the atmosphere was introduced into the model in Wolff and Bye (1996). In the surface stress solutions, it was found that a realistic momentum balance in the Antarctic channel could be obtained using form drag and surface stress coupling, without the need to involve a large (unrealistic) braking by bottom friction. In the origi- nal solutions of Wolff and Bye (1996), only the almost steady circulation was obtained. Using the same formulation, it was shown subsequently that the mesoscale eddy field associated with the almost steady solution could also be reproduced (Bye and Wolff, in press), and one of these solutions will be used to perform the dispersion experiments. The methodology of the eddy-resolving solution, as it relates to the surface velocity field, is discussed briefly below.

\section{SURFACE STRESS COUPLING}

Near the air-sea interface the surface shear stress can be represented in both fluids by an aerodynamic bulk relationship which takes account of the wavefield (Bye, 1995). In the air, we have

$$
\vec{\tau}_{\mathrm{s}}=\rho_{1} K\left|\vec{u}(z)-\left(\vec{u}_{\mathrm{o}}+\vec{u}_{L}\right)\right| \times\left[\vec{u}(z)-\left(\vec{u}_{\mathrm{o}}+\vec{u}_{\mathrm{L}}\right)\right]
$$

and in the water,

$$
\vec{\tau}_{\mathrm{s}}=\rho_{2} K\left|\left(\vec{u}_{\mathrm{o}}+\epsilon \vec{u}_{\mathrm{L}}\right)-\vec{u}(-z)\right| \times\left[\left(\vec{u}_{\mathrm{o}}+\epsilon \vec{u}_{\mathrm{L}}\right)-\vec{u}(-z)\right]
$$

where $z=0$ is the mean interfacial level, $\vec{u}$ is the fluid velocity, $\vec{u}_{0}$ is a non-wave-induced velocity, which will be called the reference velocity since it is common to both fluids, $\vec{u}_{\mathrm{L}}$ is the spectrally averaged phase velocity of the wave spectrum, and $\epsilon \vec{u}_{\mathrm{L}}$ is the spectrally integrated surface Stokes velocity, where $\epsilon=\sqrt{\rho_{1} / \rho_{2}}, K( \pm z)$ is a drag coefficient, $\rho_{1}$ and $\rho_{2}$ are respectively the densities of air and water, and $\vec{\tau}_{\mathrm{s}}$ is the interfacial shear stress (see Fig. 1). Equations (la) and (lb) are assumed to apply within a wave boundary layer which extends upwards and downwards from the interface. 


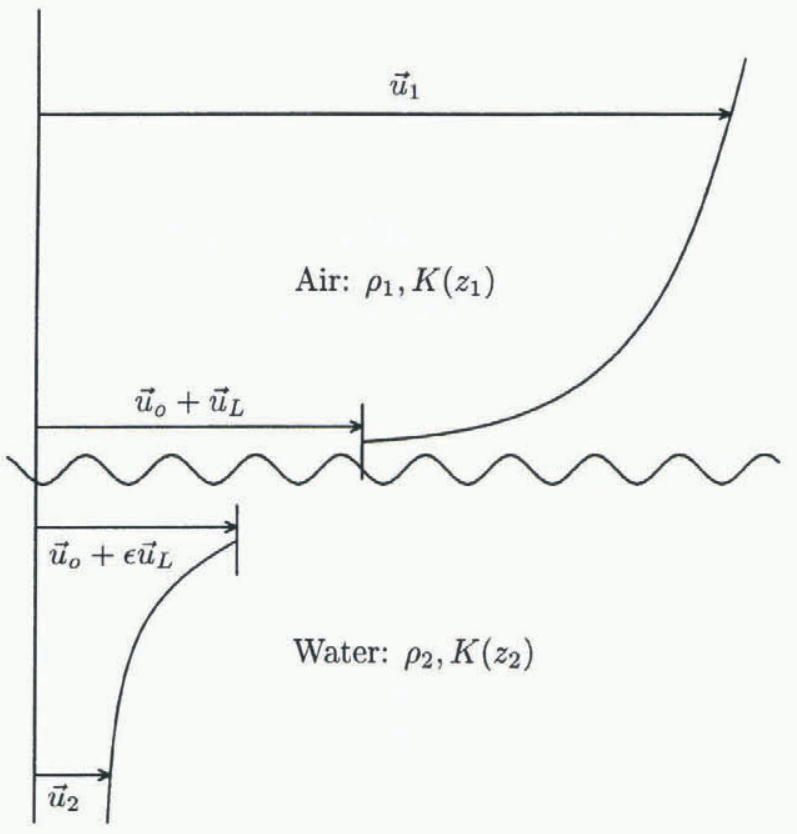

Fig. 1. Schematic picture of the inertial coupling concept. $\vec{u}_{1}$ is the surface wind, $\vec{u}_{0}$ is the reference velocity, $\vec{u}_{\mathrm{L}}$ is the phase velocity, $\vec{u}_{2}$ is the surface current, $\rho_{1}$ is the air density, $\rho_{2}$ is the water density, $\epsilon=\sqrt{\rho_{1} / \rho_{2}}$ and $K\left(z_{i}\right)(i=1,2)$ are the drag coefficients in air and water, respectively.

On equating (la) and (lb) we obtain

$$
\begin{aligned}
\vec{\tau}_{\mathrm{s}}= & \frac{\rho_{1} K}{(1+\epsilon)^{2}}\left|\vec{u}(z)-\vec{u}(-z)-(1-\epsilon) \vec{u}_{\mathrm{L}}\right| \\
& \times\left[\vec{u}(z)-\vec{u}(-z)-(1-\epsilon) \vec{u}_{\mathrm{L}}\right]
\end{aligned}
$$

and also the wave relation

$$
\epsilon\left[\vec{u}(z)-\vec{u}_{\mathrm{o}}\right]+\left[\vec{u}(-z)-\vec{u}_{\mathrm{o}}\right]=2 \epsilon \vec{u}_{\mathrm{L}} .
$$

The application of these relations at the edge of the wave boundary layer $\left(z= \pm z_{\mathrm{B}}\right)$ at which $\vec{u}_{1}=\vec{u}\left(z_{\mathrm{B}}\right)$ is the surface wind, and $\vec{u}_{2}=\vec{u}\left(-z_{\mathrm{B}}\right)$ is the surface current is considered in the situation in which the time mean reference velocity is

$$
\widetilde{\vec{u}}_{\mathrm{o}}=0
$$

which is appropriate for the Earth reference frame. Equation (4) is assumed to be valid for a short-period averaging of the wind, and a long-period averaging of the current, for which Equation (3) (applied at the edge of the wave boundary layer) reduces to the expression

$$
2 \epsilon \vec{u}_{\mathrm{L}}=\epsilon \widetilde{\vec{u}}_{1}+\widetilde{\vec{u}}_{2}
$$

which predicts the surface Stokes drift $\left(\epsilon \vec{u}_{\mathrm{L}}\right)$ in terms of $\widetilde{\vec{u}_{1}}$ and $\widetilde{\vec{u}}_{2}$. In a similar manner, the surface shear stress in the Earth reference frame $\widetilde{\vec{\tau}}_{\text {so }}$ is given by the relation,

$$
\begin{aligned}
\widetilde{\vec{\tau}}_{\mathrm{so}}= & \frac{\rho_{1} K}{(1+\epsilon)^{2}}\left|\vec{u}_{1}-\vec{u}_{2}-(1-\epsilon) \vec{u}_{\mathrm{L}}\right| \\
& \times\left[\vec{u}_{1}-\vec{u}_{2}-(1-\epsilon) \vec{u}_{\mathrm{L}}\right] .
\end{aligned}
$$

$\widetilde{\vec{\tau}}_{\text {so }}$, with $\vec{u}_{\mathrm{L}}$ given by Equation (5), is used to drive the oceanic circulation.

The solution predicts the current field $\left(\vec{u}_{2}\right)$. From this prognosis, two physically important additional velocity fields can be determined diagnostically.

First, the surface velocity $\left(\vec{u}_{\mathrm{w}}\right)$ which consists of the sum of the fluctuation in reference velocity $\left(\vec{u}_{0}-\widetilde{\vec{u}}_{\mathrm{o}}\right)$ and the sur- face Stokes drift $\left(\epsilon \vec{u}_{\mathrm{L}}\right)$ is obtained, which on using Equations (3) and (5) can be expressed as follows:

$$
\vec{u}_{\mathrm{w}}=\epsilon \vec{u}_{\mathrm{L}}+\frac{\vec{u}_{2}-\widetilde{\vec{u}}_{2}}{1+\epsilon}+\frac{\epsilon\left(\vec{u}_{1}-\widetilde{\vec{u}}_{1}\right)}{1+\epsilon}
$$

which is the sum of three terms: the surface Stokes drift, a fluctuation velocity due to the current, and a fluctuation velocity due to the wind. $\vec{u}_{\mathrm{w}}$ is applicable below the rotational turbulent interfacial layer which connects the two fluids (Bye, 1988a).

Secondly, we can determine diagnostically the surface drift velocity $\left(\vec{u}_{\mathrm{s}}\right)$ which is the vector which connects the two fluids through the turbulent interfacial layer. $\vec{u}_{\mathrm{s}}$ occurs at $z=0$ at which the velocities in the two fluids are equal, and on applying Equation (3) we obtain (Kraus, 1977)

$$
\vec{u}_{\mathrm{s}}=\frac{\epsilon \vec{u}_{1}+\vec{u}_{2}}{1+\epsilon}
$$

which on using Equations (3) and (5) can also be expressed in the form

$$
\vec{u}_{\mathrm{s}}=\frac{2 \epsilon \vec{u}_{\mathrm{L}}}{1+\epsilon}+\frac{\vec{u}_{2}-\widetilde{\vec{u}}_{2}}{1+\epsilon}+\frac{\epsilon\left(\vec{u}_{1}-\widetilde{\vec{u}}_{1}\right)}{1+\epsilon} .
$$

Equation (3) indicates, since $\epsilon \ll 1$, that the turbulent interfacial layer approximately doubles the contribution to the total velocity due to the irrotational wavefield. The contributions due to the wind and current, however, are unchanged. $\vec{u}_{\mathrm{s}}$ is the velocity which would be expected to guide surface films. It is also possible that drift cards would travel at this velocity unless wave breaking causes the cards to temporarily lose contact with the water surface.

The three velocity fields $\left(\vec{u}_{2}, \vec{u}_{\mathrm{w}}\right.$ and $\left.\vec{u}_{\mathrm{s}}\right)$ are used to disperse simulated groups of particles.

The particular quasi-geostrophic solution that is used to disperse the particles is taken from the numerical study of Bye and Wolff (in press), in which the simulation was run for $2.4 \times 10^{6}$ time-steps of 2 hours (approximately 5000 years), with a steady zonal wind and a current averaging period of 300 days. The domain consisted of an annulus of length $4000 \mathrm{~km}$ and width $1500 \mathrm{~km}$ in which a cyclic topography based on the Macquarie Ridge Complex was implemented with a mesh interval of $20 \mathrm{~km}$ (Wolff and others, 1991). A drag coefficient $K=4.8 \times 10^{-3}$ was used in Equation (6) with $\rho_{1}=1.25 \mathrm{~kg} \mathrm{~m}^{-3}$ and $\rho_{2}=1000 \mathrm{~kg} \mathrm{~m}^{-3}$ and the zonal wind field

where

$$
u_{1}(y)=\left(\frac{4 \tau^{\star}}{\rho_{1} K}\right)^{\frac{1}{2}}
$$

$$
\tau^{\star}=\tau_{0} \sin \frac{\pi y}{Y}
$$

in which $\tau_{0}=10^{-4} \mathrm{~m}^{2} \mathrm{~s}^{-2}$ and $Y$ is the width of the channel. Full details of the solution are given in Bye and Wolff (in press). Figure 2a, which shows the instantaneous solution in the upper layer at the end of the integration, indicates the level of eddy activity, and Figure $2 b$ shows the corresponding Stokes surface drift streamfield, obtained diagnostically from Equation (5).

A group of 200 particles was released over an area of approximately $1^{\circ}$ longitude by $2^{\circ}$ latitude $\left(90 \times 190 \mathrm{~km}^{2}\right)$ close to the centre of the model area. The particles were then advected by one of the velocity fields in Table 1 for the period indicated (3-30 years). The advection was computed with a very efficient fourth-order Runge-Kutta scheme to account correctly for the curvature of the flow fields. Every twelfth 


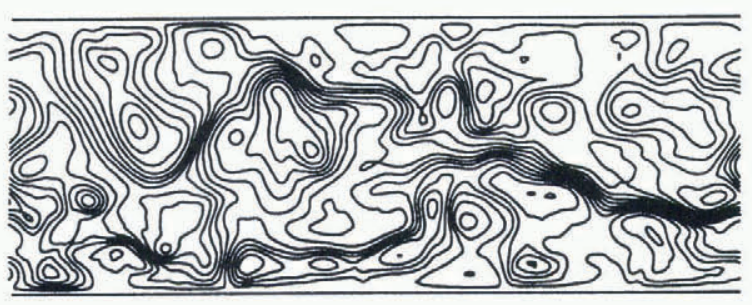

a

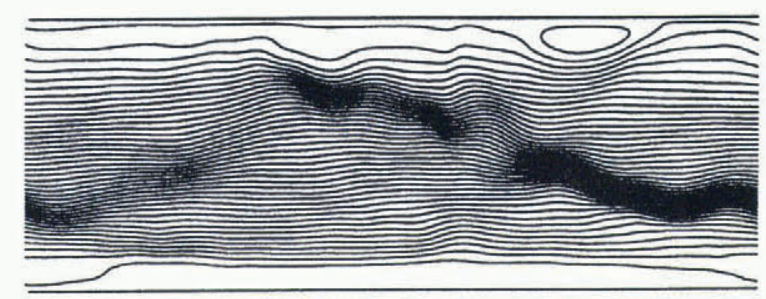

b

Fig. 2. Stream functions of the Macquarie Ridge Complex experiment MCQS300 from Bye and Wolff (in press). (a) Instantaneous stream function at the end of the integration in the upper layer (contour interval $(C I)=10^{4} \mathrm{~m}^{2} \mathrm{~s}^{-1}$ ); (b) Stokes-drift stream function $\left(C I=5 \times 10^{3} \mathrm{~m}^{2} \mathrm{~s}^{-1}\right)$.

Table 1. Model experiments

\begin{tabular}{|c|c|c|}
\hline Drift experiment & Velocity field & $\begin{array}{c}\text { Integration } \\
\text { length } \\
\text { years }\end{array}$ \\
\hline \multirow[t]{3}{*}{ Oceanic dispersion (OD) } & $\begin{array}{l}\text { Upper ocean geostrophic } \\
\text { velocity } \vec{u}_{2}\end{array}$ & 3 \\
\hline & $\begin{array}{l}\text { Surface velocity } \vec{u}_{\mathrm{w}} \\
\text { (Equation }(7))\end{array}$ & 3 \\
\hline & $\begin{array}{c}\text { Surface-drift velocity } \vec{u}_{2} \\
\text { (Equation }(8))\end{array}$ & 30 \\
\hline Atmospheric dispersion (AD) & Random fluctuations & 3 \\
\hline Coupled dispersion (CD) & $\vec{u}_{\mathrm{s}}+$ random fluctuations & 3 \\
\hline
\end{tabular}

time-step $(=1$ day), the positions of all particles were recorded (starting 1 day after the release), and at the end of the integration, the standard deviations of the particle distributions at time $(t)$ were obtained from the following formulae:

$$
\begin{aligned}
\sigma_{x}{ }^{2} & =\frac{1}{N} \sum_{n=1}^{200}\left(x_{n}-\bar{x}\right)^{2} \\
\sigma_{y}{ }^{2} & =\frac{1}{N} \sum_{n=1}^{200}\left(y_{n}-\bar{y}\right)^{2} \\
\sigma^{2} & =\sigma_{x}{ }^{2}+\sigma_{y}{ }^{2}
\end{aligned}
$$

where $(\bar{x}, \bar{y})$ are the co-ordinates of the centroid of the distribution. Trajectory plots were also obtained for a sample of 20 particles out of the 200 released.

The dispersion by wind fluctuations was investigated using a random model in which the fluctuation in wind at each gridpoint was created by a random number generator giving values between $\pm U$, where $U$ is a velocity scale. The standard deviation of the fluctuations is $\sigma_{0}=0.577 \mathrm{U}$.

\section{THEORETICAL BACKGROUND}

The release of particles paints a Lagrangian portrait of the flow field, from which its turbulent properties can be de- duced (Rodean, 1996). In this study, through the surface stress relation, we are able to examine the change in the two-dimensional turbulent field as a function of depth throughout the wave boundary layer in both fluids. The three velocity fields $\left(\vec{u}_{2}, \vec{u}_{\mathrm{w}}\right.$ and $\left.\vec{u}_{\mathrm{s}}\right)$ reveal this evolution of turbulent properties throughout the oceanic boundary layer. Any objects of finite draught respond to a depth average between these fields and also with respect to the corresponding velocity fields in the atmospheric boundary layer, the effect of which is usually called windage. In view of the simple representation of the atmospheric velocity used in this model in which "we have in reality supposed that we can divide the phenomenon into two parts, one (random) in which the discontinuity of the events taking place is essential while in the other (deterministic) it is trivial and can be ignored" (Chandrasekhar, 1943), windage is omitted in this study.

At each level, the interpretation of the results is based on the definition of the diffusion coefficient (Taylor 1921),

$$
K_{i}=\frac{1}{2} \frac{\mathrm{d}}{\mathrm{d} t} \sigma_{i}{ }^{2} \quad(i=1,2)
$$

where $K_{1}$ and $K_{2}$ are respectively the longitudinal and transverse coefficients. For a power law relation,

$$
\sigma_{i}^{2}=A_{i} t^{m_{i}}
$$

we obtain

$$
K_{i}=A_{i}{ }^{q_{i}} c_{i} t^{b_{i}}=c_{i}\left(\sigma_{i}{ }^{2}\right)^{q_{i}}
$$

where

$$
c_{i}=1 / 2 m_{i} A_{i}{ }^{1 / m_{i}}, b_{i}=\left(m_{i}-1\right) \text { and } q_{i}=\left(m_{i}-1\right) / m_{i} \text {. }
$$

Two important special cases of Equation (16) are:

(i) Brownian motion $\left(m_{i}=1\right)$ for which $b_{i}=q_{i}=0$, and $K_{i}=$ constant, $\sigma_{i}{ }^{2} \sim t$ which is the condition for an unbounded random walk.

(ii) Inertial $\left(m_{i}=3\right)$ for which $b_{i}=2, q_{i}=2 / 3$, and $K_{i} \sim \sigma_{i}{ }^{4 / 3}, \sigma_{i}{ }^{2} \sim t^{3}$ which applies for an inertial subrange in which dissipation is negligible.

A comparison between the numerical experiments and a power law representation is not straightforward, due to (i) an initial adjustment period after the particles were released, and (ii) the boundary effects of the channel walls which become significant after long transit times.

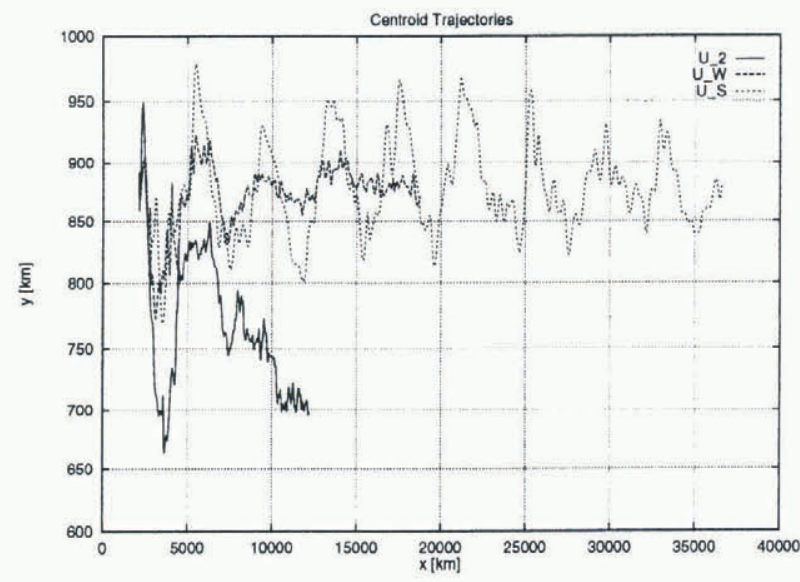

Fig. 3. Oceanic dispersion. Trajectories of the centroid of the particle distributions over a period of 3 years for the velocity fields $\vec{u}_{2}, \vec{u}_{\mathrm{w}}$ and $\vec{u}_{\mathrm{s}}$. 

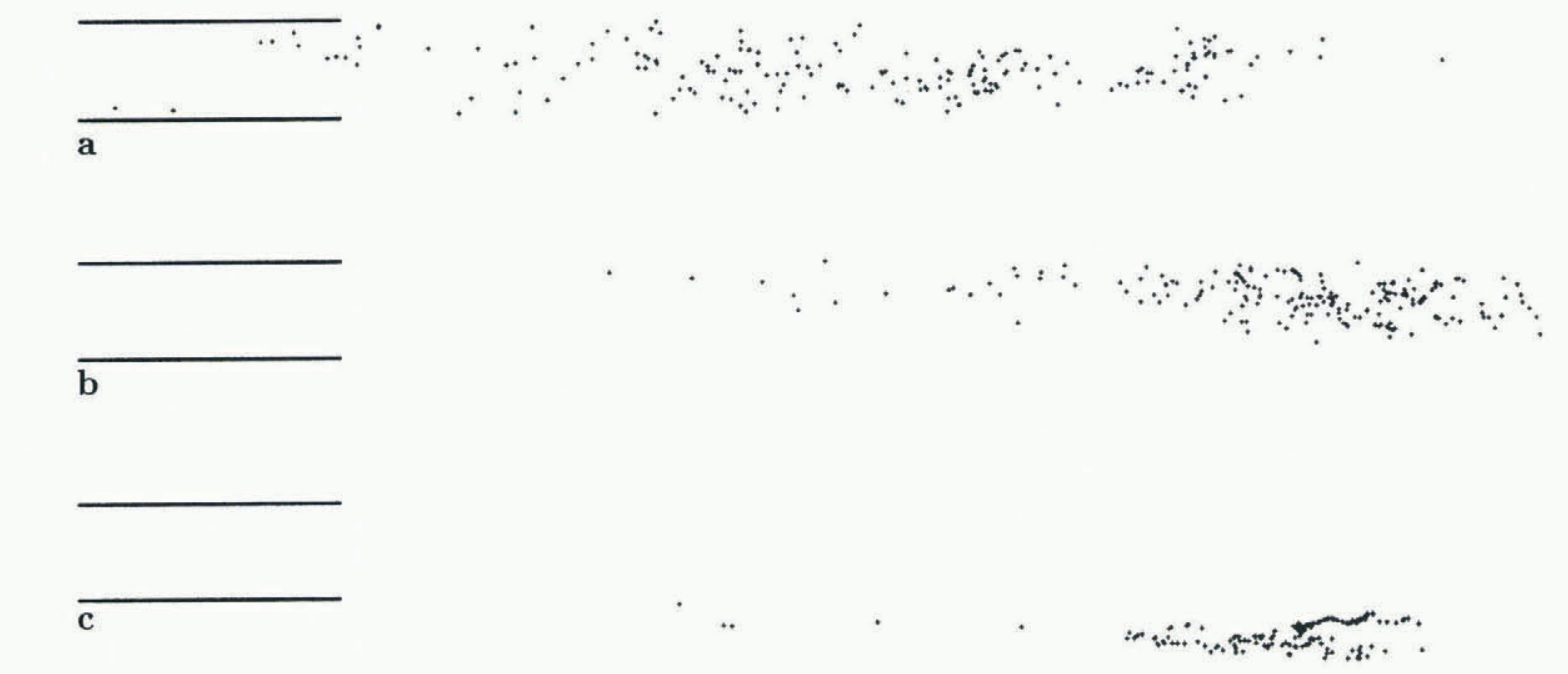

Fig. 4. Particle clouds at the end of the dispersion experiments for the velocity fields $(a) \vec{u}_{2},(b) \vec{u}_{\mathrm{w}}$ and $(c) \vec{u}_{\mathrm{s}}$. The particles have been tracked for 3 years, and in $(c)$ the tracks after 18 months have been continued one channel width below the first 18 months (to allow the same scale as in $(a)$ and $(b)$ ). The two parallel lines to the left indicate the channel dimensions. See Figure 5 for the actual tracks of a sub-sample of particles.

In the channel, the longitudinal and transverse diffusion almost correspond with zonal and meridional diffusion, respectively (see Fig. 3), and in the discussion of the results we assume that ${\sigma_{x}}^{2}=\sigma_{1}{ }^{2}$ and $\sigma_{y}{ }^{2}=\sigma_{2}{ }^{2}$ (with 1 and 2 denoting the principal axes of the diffusion ellipsoid).

\section{RESULTS OF THE DISPERSION EXPERIMENTS}

The three series of dispersion experiments (Table 1) will be called (i) oceanic dispersion (OD) in which the fluctuations in the surface wind are zero, (ii) atmospheric dispersion (AD) in which only dispersion due to the wind fluctuations is considered, and (iii) coupled dispersion (CD) in which both diffusive processes are included. The three series will be considered in turn.

\subsection{Oceanic dispersion (OD)}

Figure 3 shows the progression of the centroids of the groups of particles released into the three velocity fields. It is appar- ent that the zonal propagation of the centroid for $\vec{u}_{\mathrm{s}}$ is about twice that of $\vec{u}_{\mathrm{w}}$ (see Equations (7) and (9)), and that of $\vec{u}_{2}$ is about $60 \%$ of $\vec{u}_{\mathrm{w}}$, but until about $8000 \mathrm{~km}$ the three trajectories are fairly well correlated. Subsequently, the centroid of $\vec{u}_{2}$ tends to a stationary limit with the onset of a wellmixed state. The trajectory of the centroid for $\vec{u}_{\mathrm{s}}$, however, indicates that the particle group remains coherent throughout the experiment, i.e. for about eight revolutions of the model Antarctic Channel.

The corresponding particle clouds are shown in Figure 4. There is a strong anisotropy in the distributions. Figure 5 shows the tracks of a sample of 20 particles out of the 200 released; there is an obvious transition from the "spaghetti diagram" of the $\vec{u}_{2}$ field through to a "braided pattern" for the $\vec{u}_{\mathrm{s}}$ field. The particles in the $\vec{u}_{2}$ dispersion experiment are also clearly affected by the southern boundary.

The statistics of the dispersion are shown in Figures 6 and 7 . In all the experiments there is an extended initial period of adjustment of about 4 months $\left(10^{7} \mathrm{~s}\right)$, after which the simulated data are fitted approximately by power law

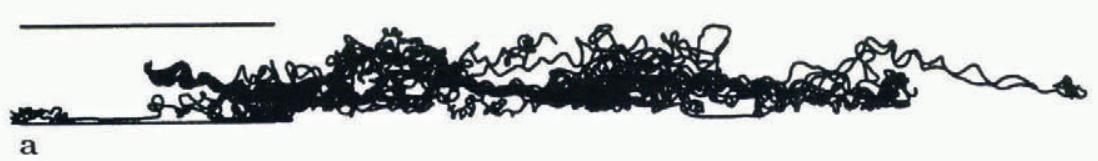

a
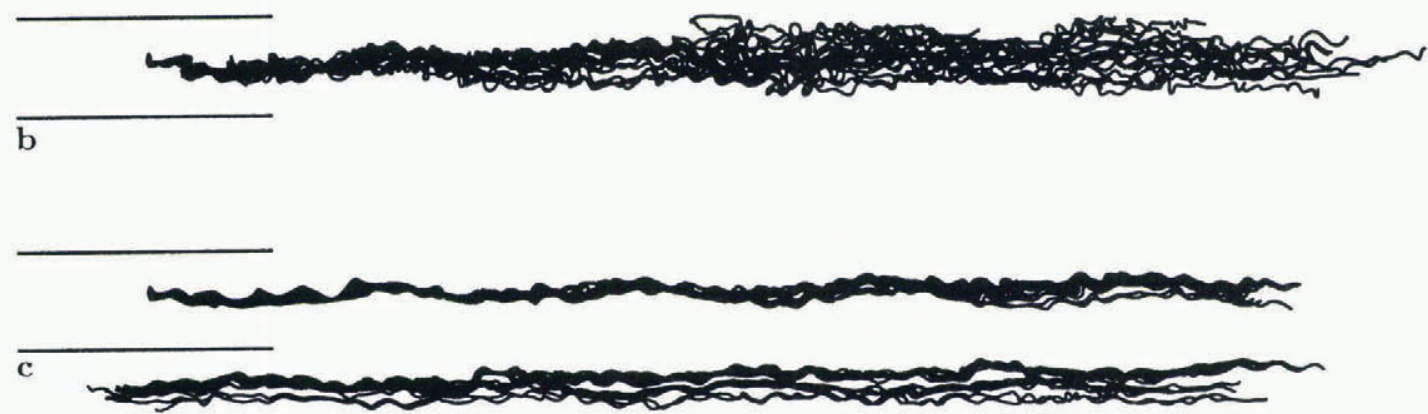

Fig. 5. Trajectories of a sample of 20 particles released into the velocity fields $(a) \vec{u}_{2},(b) \vec{u}_{\mathrm{w}}$ and $(c) \vec{u}_{\mathrm{s}}$. The particles have been tracked for 3 years, and in (c) the tracks after 18 months have been continued one channel width below the first 18 months (to allow the same scale as in ( $a$ ) and (b)). The two parallel lines to the left indicate the channel dimensions. 


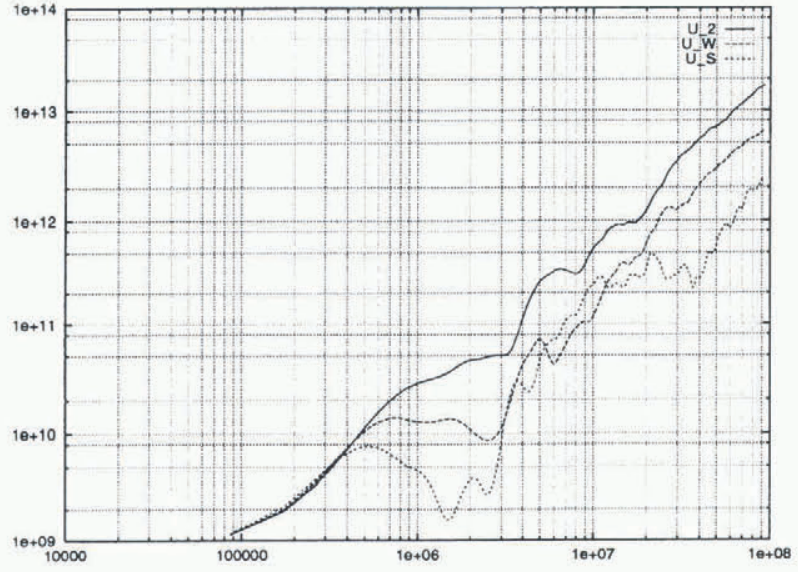

Fig. 6. The variance $\sigma_{x}{ }^{2}$ of the longitudinal dispersion for oceanic dispersion in the velocity fields $\vec{u}_{2}, \vec{u}_{\mathrm{w}}$ and $\vec{u}_{\mathrm{s}}$. The variance (ordinate) is given in units of $m^{2}$, and the time ( $a b$ scissa) in units of s.

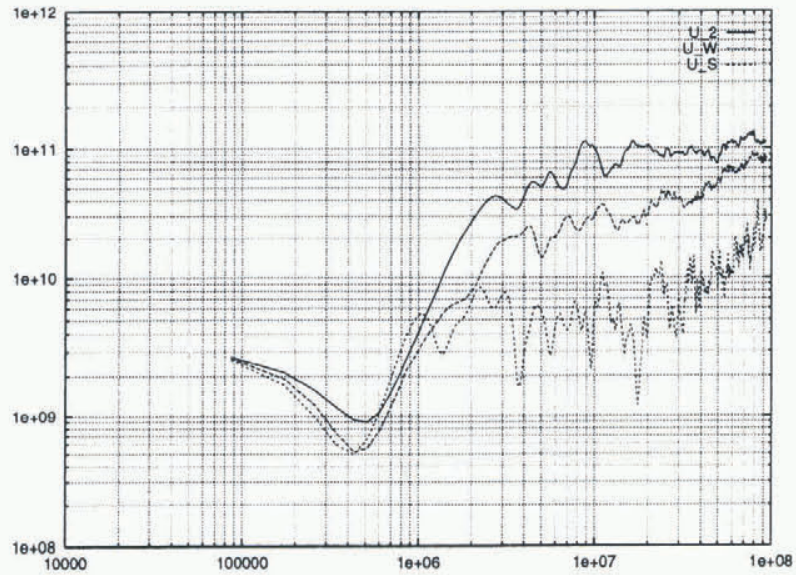

Fig. 7. The variance $\sigma_{y}{ }^{2}$ of the transverse dispersion for oceanic dispersion in the velocity fields, $\vec{u}_{2}, \vec{u}_{\mathrm{w}}$ and $\vec{u}_{\mathrm{s}}$. The variance (ordinate) is given in units of $\mathrm{m}^{2}$, and the time (abscissa) in units of s.

representations. For longitudinal dispersion the exponent $\left(m_{1}\right)$ in Equation (16) is about 1.5, for the $\vec{u}_{2}$ and $\vec{u}_{\mathrm{w}}$ fields, whereas it has increased to about 2.5 for the $\vec{u}_{\mathrm{s}}$ field. For the transverse dispersion, on the other hand, $m_{2}$ is very small in all experiments, in part due to boundary effects. The results, however, give the strong impression that in the absence of lateral boundaries the dispersion would still be strongly anisotropic.

\subsection{Atmospheric dispersion (AD)}

The dispersion due to wind fluctuations only is almost isotropic (Fig. 8), and for $U=5 \mathrm{~m} \mathrm{~s}^{-1}$ it is confined centrally within the channel. The zonal and meridional statistics are similar (Fig. 9), and the exponents tend to the random walk value, $m_{i} \sim 1$.

\subsection{Coupled dispersion (CD)}

The structure of simulations including both diffusive processes was found to be very similar to that with OD only. The only difference was that the levels of variance were somewhat elevated. Figure 10 is an extended run (for
30 years) with the $\vec{u}_{\mathrm{s}}$ field which shows the total variance $\left(\sigma^{2}\right)$ with and without AD.

There are three notable features: (i) the power law slope is reasonably stable over the longer interval; (ii) the total variance $\left(\sigma^{2}\right)$ is systematically greater for the CD solution compared with the OD solution; and (iii) the total variance $\left(\sigma^{2}\right)$ is almost identical to the longitudinal variance $\left(\sigma_{x}^{2}\right)$

(Fig. 6) for the OD solution.

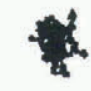

Fig. 8. Trajectories of a sample of 20 particles dispersed by random wind fluctuations with a velocity scale of $U=5 \mathrm{~ms}^{-1}$.

\section{DISCUSSION}

The following conclusions may be drawn from these experiments.

(a) Since the average eastward progression of objects in the Circumpolar Channel guided by the surface current $\left(\vec{u}_{2}\right)$ and by the surface velocity $\left(\vec{u}_{\mathrm{w}}\right)$ is similar (Fig. 3 ), it is predicted that objects with a range of draughts would travel at about the same average speed (excluding effects of windage) in agreement with field observations (Lutjeharms and others, 1988; Large and Van Loon, 1989). The "spaghetti diagram" (Fig. 5a) is typical of observations of satellite-tracked buoy trajectories. Figure 3 shows that surface drift can remain as a coherent pulse, possibly for a few global rotations, as is suggested by longterm drift-card experiments (Bye, 1988b), even though the cards may have been tumbled by wave action.

(b) The dispersion process is strongly anisotropic (see Figs 6 and 7). In these simulations, this behaviour clearly is not attributable to the shear effect, but arises from the anisotropy of the velocity field (Fig. 2a). The effects of this anisotropy characterise the thermohaline structure of the Southern Ocean.

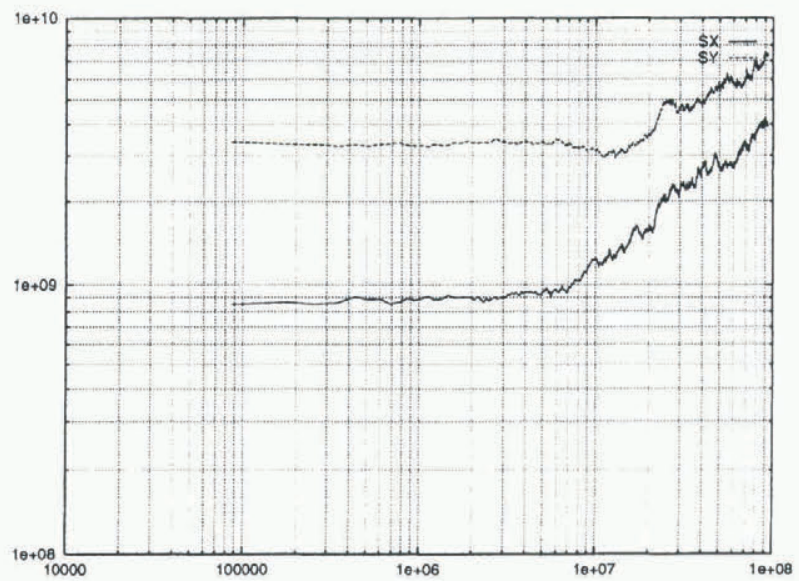

Fig. 9. The variance $\sigma_{x}^{2}$ and $\sigma_{y}^{2}$ for atmospheric dispersion with a velocity scale, $U=5 \mathrm{~ms}^{1}$. The variance (ordinate) is given in units of $m^{2}$, and the time (abscissa) in units of s. 


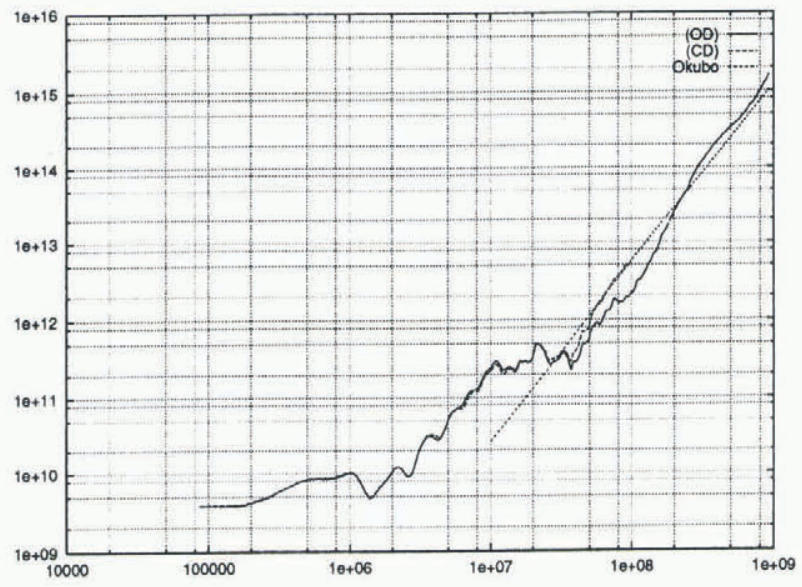

Fig. 10. The total variance $\left(\sigma^{2}\right)$ for oceanic dispersion over an extended period (30 years), and for coupled dispersion with $\vec{u}_{\mathrm{s}}$. Indicated is the power law relation for observed diffusion from Okubo (1971), where $\sigma^{2}=A\left(t^{2.34}\right)$ with $A=1.08 \times 10^{-6}$. The variance (ordinate) is given in units of $\mathrm{m}^{2}$, and the time (abscissa) in units of s.

(c) The AD experiment suggests that $\mathrm{OD}$ dominates in the Southern Ocean. This conclusion, however, would need to be tested using a more realistic atmospheric synoptic forcing.

(d) The most interesting conclusion is that the inertial character of the turbulence which causes the dispersion increases as the sea surface is approached. The simulated power law behaviour for the surface-drift $\left(\vec{u}_{\mathrm{s}}\right)$ field (Fig. $10)$ is in remarkable agreement with observed surfacedrift dispersion (Okubo, 1971), in both energy and slope. There is no a priori reason to anticipate that the inertial power law slope of 3 should be obtained. In reality, the coupled system at the sea surface is probably sub-inertial, and even less inertial at depth.
Another way of looking at the difference in the dispersion between the levels is that the expressions for $\vec{u}_{\mathrm{s}}, \vec{u}_{\mathrm{w}}$ and $\vec{u}_{2}$ consist of a steering velocity and a turbulent fluctuation. The steering velocity for $\vec{u}_{\mathrm{S}}$ is $2 \epsilon \vec{u}_{\mathrm{L}} /(1+\epsilon)$ (Equation (9)), and for $\vec{u}_{\mathrm{w}}$ it is $\epsilon \vec{u}_{\mathrm{L}}$ (Equation (7)); similarly it is $2 \epsilon \vec{u}_{\mathrm{L}}-\epsilon \widetilde{\vec{u}}_{1}$ for $\vec{u}_{2}$, at which level the fluctuation due to the wind is absent, and that due to the current is just $\left(\vec{u}_{2}-\widetilde{\vec{u}}_{2}\right)$. The steering velocity increases towards the sea surface, especially between $\vec{u}_{\mathrm{w}}$ and $\vec{u}_{\mathrm{s}}$, and this has the effect of increasing the exponent $\left(m_{1}\right)$ in the dispersion diagram.

\section{REFERENGES}

Bye, J. A. T. 1988a. The coupling of wave drift and wind velocity profiles. 7. Mar. Res., 46(3), 457-472.

Bye, J. A. T. 1988b. Drift cards in the Southern Ocean and beyond (1972-1988). Adelaide, South Australia, Flinders University. Flinders Institute of Atmospheric and Marine Science. (Cruise Report 14.)

Bye, J. A. T. 1995. Inertial coupling of fluids with large density contrast. Phys. Lett., Ser. A, $202(2), 222-224$.

Bye, J. A.T. and J. -O. Wolff. In press. On atmosphere-ocean momentum exchange in general circulation models. 7. Phys. Oceanogr.

Chandrasekhar, S. 1943. Stochastic problems in physics and astronomy. Rev. Mod. Phys., 15, 1-89,

Kraus, E. B. 1977. Ocean surface drift velocities. 7. Phys. Oceanogr., 7(4), 606-609.

Lange, W. G. and H. van Loon. 1989. Large scale, low frequency variability of the 1979 FGGE surface buoy drifts and winds over the Southern Ocean. F. Phys. Oceanogr., 19(2), 216-232.

Lutjeharms, J. R. E., L.V. Shannon and L. J. Beckman. 1988. On the surface drift of the Southern Ocean. 7. Mar. Res., 46(2), 267-279.

Okubo, A. 1971. Oceanic diffusions diagrams. Deep-Sea Res., 18(8), 789-802.

Rodean, H. C. 1996. Stochastic Lagrangian models of turbulent diffusion. Meteorol. Monogr., 26.

Taylor, G. I. 1921. Diffusion by continuous movements. Proc. Lond.Math. Soc., Ser. 2, 20, 196-211.

Wolff, J. -O. and J. A. T. Bye. 1996. Inertial surface stress coupling in a twolayer quasi-geostrophic channel model. Ocean Modelling, 112, 6-8.

Wolff, J. -O., E. Maier-Reimer and D. J. Olbers. 1991. Wind-driven flow over topography in a zonal $\beta$-plane channel: a quasi-geostrophic model of the Antarctic Circumpolar Current. F. Phys. Oceanogr., 21 (2), 236-264. 